\title{
Método para la determinación de cloruros en pequeñas concentraciones
}

CESAR DEL OLMO RODRIGUez, Dr. en Ciencias Químicas

IETCC

\section{N T R O D U C C I O N}

La determinación del contenido de cloruros de los materiales de construcción es una práctica frecuente en un laboratorio dedicado al estudio y control de calidad de estos materiales, cuyo valor es necesario conocer por varias razones; por ejemplo en las materias primas de la industria del cemento, para prevenir de antemano la formación de pegaduras en el horno ; en los componentes del hormigón, para evitar la posible corrosión de sus armaduras; o en el propio hormigón, por igual motivo, para dictaminar a posteriori las causas de la corrosión de sus alambres. El método de análisis que se describe a continuación da una respuesta válida al problema planteado.

\section{OBJETO}

El presente método de análisis tiene por objeto valorar pequeños contenidos (trazas) de cloruros. Este método tiene pues aplicación para la determinación de este constituyente $\left(\mathrm{Cl}^{-}\right)$en aquellos materiales donde su presencia es por lo general pequeña, tal es el caso de las materias primas para la industria del cemento (calizas, margas, arcillas, etc.) y de los productos intermedios de su fabricación (crudos y harinas), así como del clínker, cemento, yeso, hormigón, áridos para la construcción, etc. $\left({ }^{*}\right)$.

A los efectos de este método y por su pequeña importancia, en el valor de los cloruros se incluyen los contenidos de yoduros y de bromuros presentes en los materiales analizados.

\section{FUNDAMENTO DEL METODO}

El método se basa en la medida de la turbidez producida por la precipitación del cloruro de plata $(\mathrm{ClAg})$ en solución acidulada con ácido nítrico.

(*) Es posible valorar con exactitud en estos materiales contenidos de cloruros de hasta 0,4 ó $0,6 \%$ tomando las proporciones de muestra adecuadas para ello. Cuando la concentración de cloruros en la muestra analizada es superior a 0,50 ó $0,60 \%$, puede alcanzarse una exactitud aceptable valorando directamente este componente por el método de Mohr, de mayor simplicidad que el presente método descrito, ya que para estos órdenes de magnitud, en general, no es necesario operar con la sensibilidad que puede conseguirse con este método. 


\section{ALCANCE}

Por ser la turbidez del cloruro de plata que se mide en este método función lineal de la concentración de cloruros únicamente para pequeños contenidos de cloruros, el método es sólo aplicable cuando la solución de la muestra, preparada según el procedimiento que se describe en el apartado 6.1., contiene una concentración de ion cloro igual o menor que $0,4 \mathrm{mg} / 100 \mathrm{ml}\left(^{*}\right)$.

\section{APARATOS NECESARIOS}

- Machacadora y molino (**).

— Tamiz con luz de malla de 0,200 mm (900 mallas $\left./ \mathrm{cm}^{2}\right) \quad(* *)$.

- Balanza analítica de precisión.

- Estufa regulada a $105^{\circ}-110^{\circ} \mathrm{C}$.

- Crisol de platino $(* * *)$.

- Baño maría.

- Baño termostatado regulable a $40^{\circ} \pm 1^{\circ} \mathrm{C}$.

- Nefelómetro con sus accesorios.

- Pipetas aforadas de 1, 2, 5, 10 y $20 \mathrm{ml}$.

- Matraces aforados de 100, 500 y $1.000 \mathrm{ml}$.

- Vasos de precipitado de 100, 250 y $600 \mathrm{ml}$.

- Embudos, etc.

\section{REACTIVOS NECESARIOS}

- Carbonato sódico anhidro (****).

- Disolución de ácido nítrico $1: 1$.

- Disolución de nitrato de plata al $1 \%$. Se prepara por disolución de $1 \mathrm{~g}$ de $\mathrm{NO}_{3} \mathrm{Ag}$ en $100 \mathrm{ml}$ de agua destilada.

- Papel indicador de $\mathrm{pH}$.

NOTA: Es indispensable utilizar en todas las operaciones requeridas en el método (disoluciones, lavado de residuos, etc.) agua destilada exenta de cloruros, debiéndose rechazar, por lo tanto, el agua destilada que dé la menor opalescencia al añadirle, en medio nítrico, unas gotas de disolución de nitrato de plata al $1 \%$.

(*) Esto equivale a decir que el método es válido siempre que la lectura de la turbidez de la solución preparada cor la muestra-incógnita sea inferior a la lectura de la turbidez del patrón más concentrado que se toma como 100.

(**) Estos instrumentos son necesarios en el caso del análisis de materiales no pulverulentos.

(****) La utilización del crisol de platino y del fundente $\left(\mathrm{CO}_{3} \mathrm{Na}_{2}\right)$ se precisa sólo para el análisis de ciertos materia!es (arcillas, margas, etc.); véase, no obstante, lo indicado sobre su empleo en el apartado 6.1 2.

(****) El empleo de este fundente se precisa sólo para el análisis de ciertos materiales (arcillas, margas, etc.); véase, no obstante, lo indicado sobre su utilización en el apartado 6.1.2. 


\subsection{Preparación de la serie-patrón de cloruros}

\subsubsection{Disolución-patrón matriz}

Disolver 0,824 g de ClNa p.a. en $400 \mathrm{ml}$ de agua destilada y transferir esta disolución a un matraz aforado de $500 \mathrm{ml}$, enrasándolo con agua destilada (de esta forma la solución contiene $0,001 \mathrm{~g}$ de $\mathrm{Cl}^{-} / \mathrm{ml}$ ).

\subsubsection{Disolución-patrón de cloruros}

Tomar $5 \mathrm{ml}$ de la "Disolución-patrón matriz", preparada según ha quedado descrito en el apartado 5.1.1., y diluir a $500 \mathrm{ml}$ con agua destilada en un matraz aforado de esta capa-

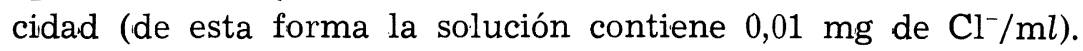

\subsubsection{Disolución de iones concurrentes $\left({ }^{*}\right)$}

La "Disolución de iones concurrentes" se prepara por mezcla de las soluciones A y B, cuya preparación se describe a continuación, teniendo en cuenta las indicaciones señaladas seguidamente.

\section{Solución A}

Disolver en unos $400 \mathrm{ml}$ de agua destilada contenida en un vaso de precipitado de $600 \mathrm{ml}$ de capacidad, las siguientes cantidades de las sales que se indican a continuación:

\begin{tabular}{cc} 
Peso & \multicolumn{1}{c}{ sal } \\
\hline $0,736 \mathrm{~g}$ & $\left(\mathrm{NO}_{3}\right)_{3} \mathrm{Al} .9 \mathrm{H}_{2} \mathrm{O}$ p.a. \\
$0,506 \mathrm{~g}$ & $\left(\mathrm{NO}_{3}\right)_{3} \mathrm{Fe} .9 \mathrm{H}_{2} \mathrm{O}$ p.a. \\
$8,695 \mathrm{~g}$ & $\left(\mathrm{NO}_{3}\right)_{2} \mathrm{Ca} .4 \mathrm{H}_{2} \mathrm{O}$ p.a. \\
$0,636 \mathrm{~g}$ & $\left(\mathrm{NO}_{3}\right)_{2} \mathrm{Mg} \cdot 6 \mathrm{H}_{2} \mathrm{O}$ p.a.
\end{tabular}

\section{Solución B}

Disolver en unos $300 \mathrm{ml}$ de agua destilada a los que se añaden $5 \mathrm{ml}$ de $\mathrm{NO}_{3} \mathrm{H} 1: 1$, contenidos ambos líquidos en un matraz aforado de $1.000 \mathrm{ml}$ :

$$
0,558 \mathrm{ml} \text { de } \mathrm{SiO}_{3} \mathrm{Na}_{2} \text { siruposo }
$$

Por último, verter lentamente y agitando para procurar que no se forme precipitado la disolución A sobre la disolución $\mathrm{B}$ que se encuentra contenida en el matraz aforado antes indicado y enrasar éste a $1.000 \mathrm{ml}$ con agua destilada. Si la disolución no resulta transparente, debe filtrarse a través de un papel de filtro de porosidad media.

(*) Véase lo indicado en la llamada primera del apartado 6.2.1. 


\section{METODO DE ENSAYO}

\subsection{Preparación de la muestra-problema}

\subsubsection{Molienda}

Desecar la muestra en una estufa regulada a $105^{\circ}-110^{\circ} \mathrm{C}$ hasta obtener constancia de peso.

Las muestras que no tengan la finura adecuada deben molerse antes de su análisis hasta alcanzar una finura menor de 200 micras (residuo cero sobre tamiz de 900 mallas $/ \mathrm{cm}^{2}$ ). El cemento y otros materiales de similar finura no requieren esta molienda previa.

\subsubsection{Ataque y disolución}

La disolución de los cloruros de la mayoría de los materiales considerados en este trabajo puede conseguirse por medio de un ataque ácido. No obstante, existen algunos de entre estos materiales (arcillas, margas, crudos, harinas, etc.) que para lograr la total disolución de sus cloruros es necesario aplicar una fusión alcalina de la muestra; aunque puede prescindirse de dicha fusión cuando en el análisis no se requiere gran exactitud porque el error que con esta simplificación se introduce es, en general, muy pequeño.

En uno y otro caso el procedimiento que se aplica para obtener la "solución preparadaproblema" es el que se describe a continuación:

\subsubsection{Sin fusión alcalina}

Pesar $1 \mathrm{~g}$ de la muestra desecada a $105^{\circ}-110^{\circ} \mathrm{C}$, con una exactitud superior a la milésima de gramo (*).

Situar la muestra pesada en un vaso de precipitado a $100 \mathrm{ml}$ de capacidad y de forma alta, añadir $10 \mathrm{ml}$ de ácido nítrico $1: 1\left(^{* *}\right)$ y calentar en baño maría hasta completar el ataque y eliminación del dióxido de carbono de la muestra, lo que generalmente se consigue en un lapso de tiempo de unos 5 minutos; diluir seguidamente con agua destilada hasta obtener un volumen de unos $30 \mathrm{ml}$, manteniendo unos momentos el vaso de precipitado en el baño maría y a continuación hervir los líquidos unos instantes. Enfriar y filtrar la disolución a través de un papel de filtro de porosidad media (banda blanca); lavar con agua destilada el residuo que queda sobre el papel de filtro, operación que se

(*) Cuando se desconoce el orden de magnitud de los cloruros existente en la muestra analizada, por precaución se aconsieja partir de $2 \mathrm{~g}$ de muetra-problema, que se atacarán con el doble de las cantidades de reactivo señaladas en el apartado 6.1.2.1., obteniéndose al final $200 \mathrm{ml}$ de la solución preparada-problema (contienidos en un matraz aforado de esta capacidad). Los primeros $100 \mathrm{ml}$ de dicha disolución se destinan a la valoración del contenido de cloruros por el método de Mohr (determinación volumétrica con nitrato de plata en presencia de cromato potásico como indicador interno, operación que se efectúa después de separar de la solución preparada-problema los hidróxidos precipitados con una disolución de $\mathrm{NaOH}$ ), continuándose la valoración de los restantes $100 \mathrm{ml}$ de la solución preparada-problema por el presente método descrito, si el contenido de cloruros ha resultado infierior a 0,4 ó $0,5 \%$.

(**) Si la muestra analizada es de naturaleza caliza, es necesario emplear mayor cantidad de ácido nítrico $1: 1$ que la indicada porque parte del ácido se consume en neutralizar los carbonatos. En este caso, se inicia el ataque de la muestra añadiendo gota a gota la disolución de ácido nítrico $1: 1$ hasta que cesa la aparición de espuma; entonces se adiciona el exceso de $10 \mathrm{ml}$ de $\mathrm{NO}_{3} \mathrm{H} 1: 1$. 
da por terminada cuando los líquidos que filtran del lavado del residuo alcancen un valor de $\mathrm{pH}$ próximo a 7 -neutralidad- (comprobado con papel indicador de $\mathrm{pH}$ ), obteniéndose en todo el proceso un volumen total de líquidos filtrados de unos 70 a $80 \mathrm{ml}$, que se recogen en un matraz aforado de $100 \mathrm{ml}$, y por último, se añade agua destilada para enrasar dicho matraz. A esta disolución se le denomina "solución preparada-problema" $(*)$.

\subsubsection{Con fusión alcalina}

Pesar $1 \mathrm{~g}$ de la muestra desecada a $105^{\circ}-110^{\circ} \mathrm{C}$, con una exactitud superior a la milésima de gramo $(* *)$.

Introducir la muestra pesada en un crisol de platino en el que se han situado unos $5 \mathrm{~g}$ de carbonato sódico anhidro, mezclar cuidadosamente el contenido del crisol y añadir, por último, una pequeña cantidad de $\mathrm{CO}_{3} \mathrm{Na}_{2}$ para formar sobre la mezcla anterior una pequeña capa del fundente; cubrir el crisol con su tapadera e iniciar la calefacción. La calefacción se realiza calentando el crisol sobre un mechero de gran potencia, primeramente, durante unos 10 minutos, con poca llama para evitar proyecciones y salpicaduras, y, por último, con la llama graduada a su máxima potencia durante otro período de 20 minutos; una vez transcurridos los primeros 15 minutos de este segundo período se separa del crisol su tapadera y se homogeneiza la mezcla fundida, removiendo para ello el crisol sobre el mechero con ayuda de unas pinzas de platino de vástago largo. La tapadera del crisol, en primer lugar, y después el crisol con la mezcla fundida se introducen en un vaso de precipitado de $250 \mathrm{ml}$ de capacidad con unos $75 \mathrm{ml}$ de agua destilada; el vaso se tapa con un vidrio de reloj y se calienta en un baño maría hasta que se desprende del crisol la pastilla fundida; entonces se separan del vaso el crisol y su tapadera, no sin antes haber quedado limpios de todo resto de fundido, operación que se efectúa con agua destilada caliente y después con ácido nítrico 1:1. La disolución de la pastilla fundida que se ha iniciado con agua, se continua por adición de ácido nítrico $1: 1$ (que se añade poco a poco para evitar que el líquido se derrame) hasta que cesa la formación de espuma, incorporando, por ultimo, un exceso de $10 \mathrm{ml}$ de ácido nítrico $1: 1$; los líquidos se hierven después unos momentos hasta lograr la total eliminación del dióxido de carbono de la muestra fundida. Seguidamente los líquidos se filtran a través de un papel de filtro de porosidad media (banda blanca) y se lava el residuo que queda sobre el papel de filtro con agua destilada caliente hasta que los líquidos que filtran del lavado alcancen un valor de $\mathrm{pH}$ próximo a 7 (comprobado con papel indicador de $\mathrm{pH}$ ), recogiendo los líquidos filtrados en un matraz aforado de $250 \mathrm{ml}$ de capacidad, que se enrasa con agua destilada. Una vez homogeneizados los líquidos contenidos en este matraz, por agitación del mismo, se pasan $100 \mathrm{ml}$ de la disolución a un matraz aforado de esta capacidad. A esta disolución se le denomina también "solución preparada-problema" $(* * *)$.

(*) Cuando la muestra analizada contiene una concentración de ion cloruro inferior a $0,04 \%$, en el ensayo se utiliza la totalidad del la «solución preparada-problema», debiéndose, en este caso, prescindir de enrasar el matraz que contiene estos líquidos para que puedan tener cabida en el volumen de $100 \mathrm{ml}$ los reactivos $\left(2\right.$ ml de $\mathrm{NO}_{3} \mathrm{H} \quad 1: 1$ y $2 \mathrm{ml}$ de $\mathrm{NO}_{3} \mathrm{Ag}$ al $1 \%$ ) que deberán adicionarse posteriormente al matraz (véase párrafo 6.2.2.).

(**:) Véase lo indicado en la llamada primera diel apartado anterior (6.1.2.1.).

(***) Cuando la muestra analizada contiene una concentración de ion cloruro inferior a $0,10 \%$, en el ensayo se utiliza la totalidad de la «solución preparada-problema», debiéndose, en este caso, después de homogeneizar los líquidos contenidos en el matraz, separar de ellos $5 \mathrm{ml}$ medidos con pipeta aforada, para que puedan tener cabida en el volumen de $100 \mathrm{ml}$ los reactivos $\left(2 \mathrm{ml}\right.$ de $\mathrm{NO}_{3} \mathrm{H} 1: 1$ y $2 \mathrm{ml}$ de $\mathrm{NO}_{3} \mathrm{Ag}$ al $1 \%$ ) que hay que añadir posteriormente al matraz (véase el párrafo 6.2.2.), operando en estas condiciones con $95 \mathrm{ml}$ de la solución preparada-problema, lo que hay que tener en cuenta al efectuar los cálculos. 


\subsection{Preparación de las suspensiones de cloruros de plata}

NOTA: Antes de proceder a la preparación de las suspensiones de cloruro de plata (de los patrones y de la muestra incógnita) cuya técnica se describe en este apartado, es necesario tener a régimen el baño termostatado regulado a $40^{\circ} \pm 1^{\circ} \mathrm{C}$ que se precisa para realizar la valoración de cloruros según este método, y disponer de agua con hielo para las operaciones posteriores.

\subsubsection{Suspensiones-patrón de cloruro de plata}

Disponer ocho matraces aforados de $100 \mathrm{ml}$ de capacidad debidamente rotulados para su fácil identificación y verter en cada uno de ellos $20 \mathrm{ml}$ de la "disolución de iones concurrentes" (preparada según el procedimiento descrito en el apartado 5.1.3.) $\left(^{*}\right)$ y las cantidades respectivas que se indican a continuación de la "disolución-patrón de cloruros" (preparada igualmente según el procedimiento descrito en el apartado 5.1.2.) (**).

Las cantidades de la "disolución-patrón de cloruros" añadidas a los siete primeros matraces son respectivamente: $40,20,10,5,4,2$ y $1 \mathrm{ml}$, que se miden con pipetas aforadas. Al último matraz (n. ${ }^{\circ}$ ) no se le incorpora cantidad alguna de la mencionada "disolución de cloruros".

En cada uno de los ocho matraces anteriores se introducen además $2 \mathrm{ml}$ de ácido nítrico $1: 1$ y $2 \mathrm{ml}$ de nitrato de plata al $1 \%$ y, por último, se enrasan los matraces con agua destilada.

Los matraces enrasados contienen las siguientes cantidades de ion cloruro.

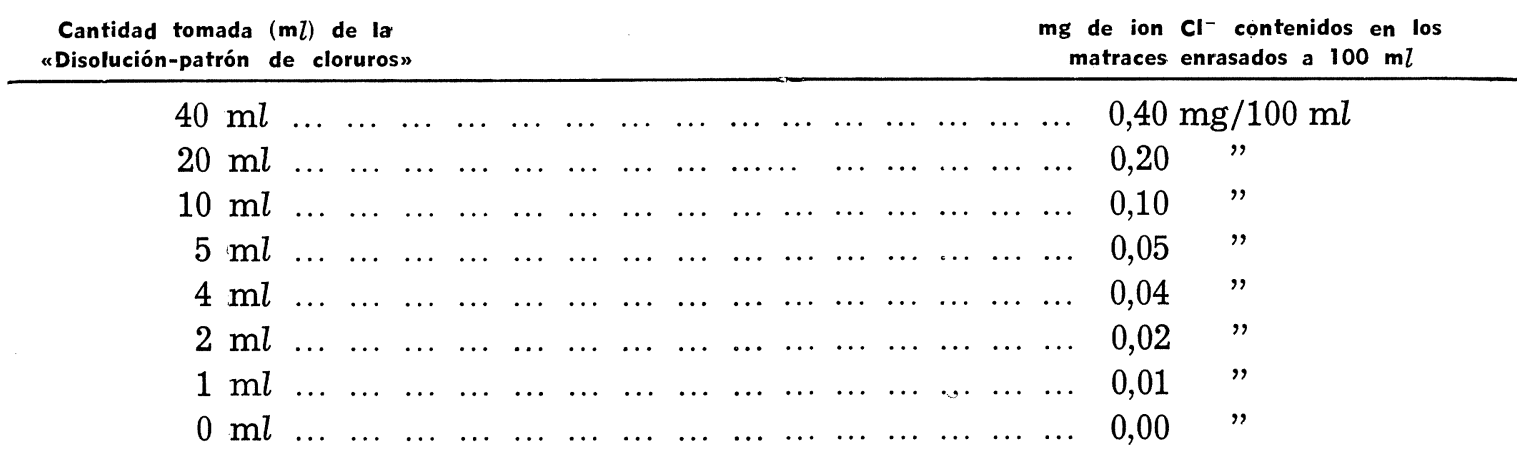

En general bastará con preparar las 5 disoluciones de $0,40,0,20,0,10,0,05$ y $0,00 \mathrm{mg}$ $\mathrm{Cl}^{-} / 100 \mathrm{ml} ; \mathrm{y}$ solamente cuando la concentración de cloruros en la muestra sea muy baja, será necesario preparar las 3 disoluciones restantes de $0,04,0,02$ y $0,01 \mathrm{mg}$ de $\mathrm{Cl}^{-} / 100 \mathrm{ml}$.

(*) Puede presclindirse de la adición de la «disolución de iones concurrentes» en análisis donde no se precisa una elevada exactitud en los resultados.

(**) Es necesario dejar transcurrir un lapso de tiempo determinado (del orden de unos 5 minutos aproximadamente) entre la preparación de cada suspehsión de cloruro de plata y la preparación de la suspensión siguilente, para que, al disponer del tiempo suficiente que nos permita realizar las operaciones indicadas en los apartados 6.3. y 7., puedan efectuarse todas las lecturas del aparato a iguales intervalos de tiempo contados a partir del momento de la adición del nitrato de plata. Los patrones de 0,00 y de $0,40 \mathrm{mg}$ de $\mathrm{Cl}^{-} / 100 \mathrm{ml}$, y precisamente en este orden, deben preparase en primer lugar porque dichos patrones son los primeros que hay que utilizar en la calibración del nefelómetro según se indica en el apartado 7. 


\subsubsection{Suspensión de la muestra-incógnita}

En otro matraz aforado de $100 \mathrm{ml}$ marcado también con la rotulación precisa, verter un cierto volumen de la "solución preparada-problema" (que se ha obtenido tras las operaciones descritas en el apartado 6.1), $2 \mathrm{ml}$ de ácido nítrico $1: 1 \mathrm{y}$. $2 \mathrm{ml}$ de nitrato de plata al $1 \%$. Por último, enrasar el matraz con agua destilada (*);

\subsection{Técnica para conseguir reproductividad en el tamaño de grano de las suspensiones de cloruro de plata}

Inmediatamente después de enrasados los matraces que contienen las suspensiones de cloruro de plata obtenidas (de los patrones y de la muestra incógnita), se invierten dichos matraces dos o tres veces para homogeneizar los líquidos en ellos contenidos.

A continuación, se introducen los matraces en un baño de agua, termostatado a la temperatura de $40^{\circ} \pm 1^{\circ} \mathrm{C}$, manteniendo dichos matraces en estas condiciones durante $30 \pm 0,5$ minutos, contados a partir del momento en que se adicionó el nitrato de plata $\left({ }^{* *}\right)$.

Transcurridos los 30 minutos, se sacan los matraces del termostato y se sumergen en agua con hielo para que alcancen rápidamente la temperatura ambiente, lo que se consigue en un período de unos 5 minutos. Una vez enfriados los matraces a la temperatura ambiente se sacan sucesivamente del agua helada y se mide su turbidez (poder de dispersión de la luz en nefelometría). Es necesario efectuar en todos los matraces las operaciones subsiguientes a la adición del nitrato de plata con ligereza y del mismo modo. La fotografía 1 muestra esta fase del análisis.

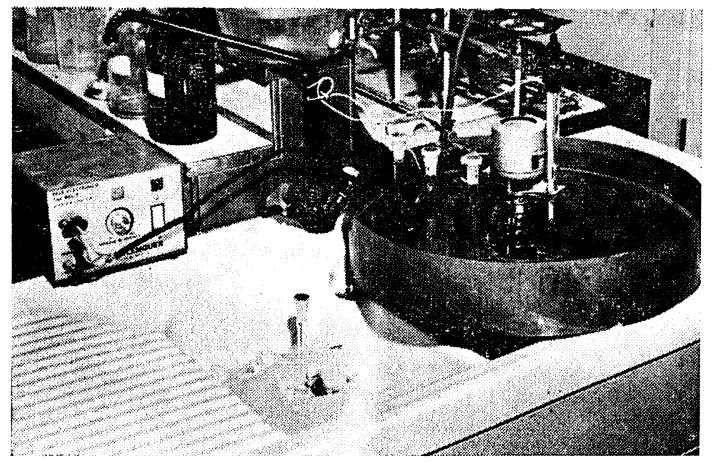

Fotografía 1.-Dispositivo para conseguir reproductividad en el tamaño de grano de las suspensiones de cloruro de flata (baño termostatado y agua con hielo).

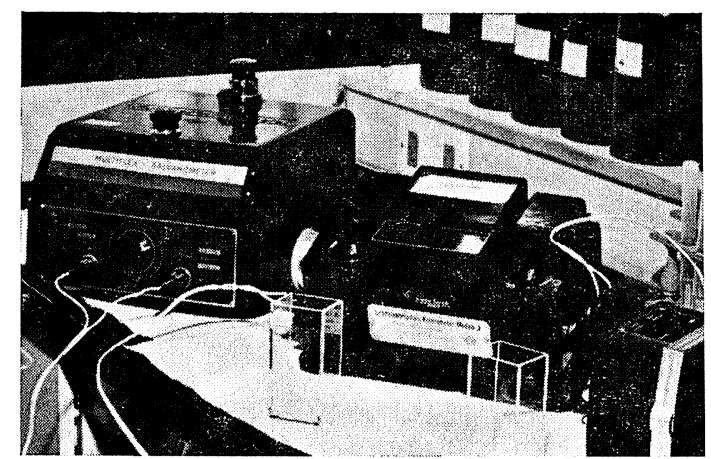

Fotografia 2.-Nefelómetro y galvanómetro «multiflex».

(*) La cantidad de la «solución preparada-problema» que debe tomarse es aquélla que proporcione un valor de la dispersión de la luz situado dentro de la escala construida con los patrones de cloruro preparados. Cuando se toma la totalidad de la «solución preparada-problema», téngase en cuenta lo indicado al pie de página de los apartados 6.1.2.1. y 6.1.2.2. referente a no enrasar el matraz que contiene esta solución para que puedan tener cabida en él los reactivos que es necesario añadir.

(*:*) De estos 30 minutos, se invierten unos 12 minutos en subir la temperatura interior del matraz desde la temperatura ambiente hasta $40^{\circ} \mathrm{C}$. 


\section{\%. MEDIDA DE LA LUZ DISPERSADA}

La medida de la luz dispersada por las distintas suspensiones de cloruro de plata preparadas se efectúa con ayuda de un nefelómetro de elevada sensibilidad, pudiendo aumentarse la sensibilidad del aparato acoplando al nefelómetro un galvanómetro del tipo "multiflex". En la fotografía 2 puede observarse un montaje de este tipo.

Antes de introducir las suspensiones de cloruro de plata en la cubeta del nefelómetro, es necesario agitar dos o tres veces los matraces que las contienen para homogeneizar los líquidos.

Se comienzan las medidas de la dispersión de la luz graduando el aparato con las suspensiones-patrón de cloruros más diluida $(0,00)$ y más concentrada $(0,40)$, con las que se imponen en la correspondiente escala del nefelómetro los valores respectivos de cero y de cien; a continuación se obtienen con las suspensiones-patrón de cloruros de concentraciones intermedias las restantes lecturas que nos servirán para trazar la curva de calibrado, y por último, se mide en la misma escala el valor de la dispersión de la luz producida por la suspensión-problema.

\section{OBTENCION DE LOS RESULTADOS}

El valor del contenido de cloruros de la muestra analizada se obtiene por extrapolación gráfica.

Para ello, se dibuja en un papel milimetrado un sistema de coordenadas, representando en el eje de ordenadas los valores de las concentraciones de cloruros y en el eje de abscisas las lecturas obtenidas en el aparato. La curva de calibrado resulta de unir en dicho sistema de coordenadas los puntos representativos de las distintas soluciones-patrón de cloruros. El valor de la concentración de cloruros de la disolución preparada-problema, es el correspondiente a la ordenada del punto en que la perpendicular trazada por el valor de su lectura en el aparato, representado en el eje de abscisas, corta a la curva de calibrado.

En el apartado 10 se incluye un ejemplo del modo cómo puede obtenerse el valor de la concentración de cloruros de la disolución preparada-problema.

Por último, el contenido de cloruros de la muestra analizada, expresado en tanto por ciento de ion cloro, se obtiene por cálculo a partir de la expresión siguiente:

donde:

$$
\text { Contenido de cloruros }\left(\mathrm{Cl}^{-}\right), \%=\frac{V \cdot y_{1}}{10 \cdot P \cdot v}
$$

$$
\begin{aligned}
V= & \text { volumen total, en } \mathrm{m} l \text {, de la "solución preparada-problema" }(*) ; \\
y_{1}= & \text { valor de la ordenada del punto (expresado en } \left.\mathrm{mg} \mathrm{Cl}^{-} / 100 \mathrm{ml}\right) \text { en que la perpendi- } \\
& \text { cular trazada por el valor de la lectura del aparato representado en el eje de abs- } \\
& \text { cisas corta a la curva de calibrado; } \\
P= & \text { peso, en g, de muestra tomada en el ensayo; } \\
v= & \text { volumen, en ml, de la anterior "solución preparada-problema" (de un total de } 100 \\
& \text { ó de } 250 \mathrm{~m} l \text { según los casos) medidos en la cubeta del nefelómetro. }
\end{aligned}
$$

(*) El valor de $V$ es de $100 \mathrm{ml}$ en el caso de una muestra disuelta con ataque ácido (nítrico) y de $250 \mathrm{ml}$ en el caso de una muestra disuelta mediante fusión alcalina. 


\section{PRECISION DE LOS RESULTADOS}

En ausencia de interferencias (*), la precisión alcanzada y las tolerancias admitidas son:

\subsection{Precisión}

Los resultados de dos ensayos no deben diferir en más de 0,005\%.

\subsection{Tolerancias}

Las medias de los resultados no deben diferir del contenido real de ion cloro en más de $\pm 0,005 \%$.

\section{EJEMPLO ACLARATORIO}

En la determinación del contenido de cloruros de una muestra de cemento, realizada por el método nefelométrico anteriormente descrito, se ha obtenido una lectura de 40 unidades en la escala del aparato para una cantidad de muestra ensayada de $1 \mathrm{~g}$, de la que se han valorado $50 \mathrm{ml}$ de la "solución preparada-problema" (enrasada a $100 \mathrm{ml}$ ). En dicha escala las lecturas obtenidas con las soluciones-patrón de cloruros han sido las siguientes:

mg de ion $\mathrm{Cl}^{-}$contenidos en lọs

$100 \mathrm{~m} l$ de las soluciones-patrón

Lectura del aparato

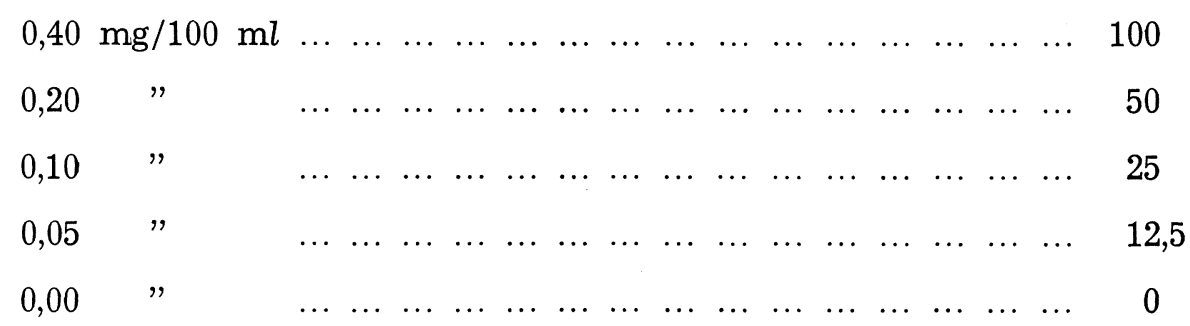

Representando los valores anteriores en un sistema de coordenadas, se obtiene la curva de calibrado trazada en la figura 1. En esta figura el contenido de ion cloro de la fracción de muestra de cemento analizada, corresponde al valor de la ordenada $\left(y_{1}\right)$ del punto de intersección $\left(P_{1}\right)$ entre la curva de calibrado y la perpendicular al punto $\left(x_{1}\right)$, representativo del valor de la lectura obtenida en el nefelómetro con la fracción de muestra analizada.

(*) Cuando el material en el que se trata de determinar la concentración de cloruros contiene una proporción apreciable de sulfuros, puede producirse la precipitación de sulfuro de plata $\left(\mathrm{SAg}_{2}\right)$ de color negro al añadir la disolución de nitrato de plata en la secuencia de operación 6.2.2. del presente método. En tales casos —raros-, esta interferencia puede ser eliminada llevando a sequedad en una cápsula, al baño maría, la solución preparada-problema (obtenida en la secuencia 6.1.2.); añadiendo al residuo seco resultante $0,5 \mathrm{ml}$ de agua oxigenada al $30 \%$ y redisolviendo el residuo con unos $80 \mathrm{ml}$ de agua destilada, con lo que se tiene la nueva «solución preparada-problema» libre de esta interferencia. 


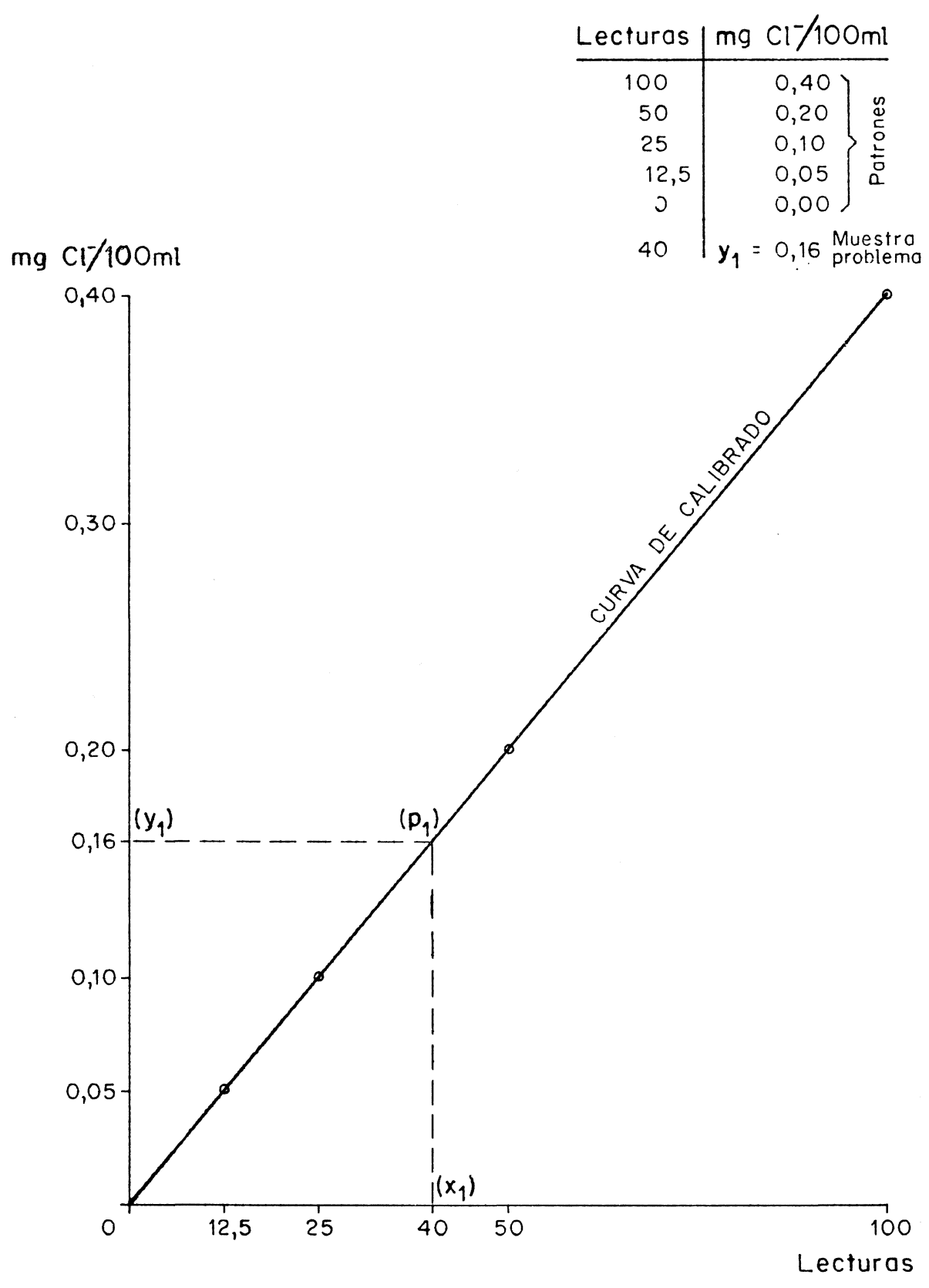

Fig. 1

Para expresar el contenido de cloruros de la muestra de cemento examinada en tanto por ciento, se aplica la expresión [1] del apartado 8, habiendo resultado en este caso un valor de $y_{1}=0,16 \mathrm{mg}$ para unos parámetros de $V=100 \mathrm{ml}, P=1 \mathrm{~g}$ y $v=50 \mathrm{ml}$.

Por lo tanto, el

Contenido de cloruros $\left(\mathrm{Cl}^{-}\right), \%=\frac{V \cdot y_{1}}{10 \cdot P \cdot v}=\frac{100 \times 0,16}{10 \times 1 \times 50}=0,032 \pm 0,005 \%$. 